\title{
Correction to: Gestational chronic intermittent hypoxia induces hypertension, proteinuria, and fetal growth restriction in mice
}

\author{
Cuilian Weng ${ }^{1} \cdot$ Long Huang ${ }^{1} \cdot$ Hangwei Feng ${ }^{1} \cdot$ Quanying $\mathrm{He}^{2} \cdot$ Xingsheng Lin $^{1} \cdot$ Tingting Jiang $^{3} \cdot$ Jian Lin $^{1}$. \\ Xincai Wang ${ }^{1} \cdot$ Qinghua Liu ${ }^{4}$
}

Published online: 12 February 2022

○) Springer Nature Switzerland AG 2022

\section{Correction to: Sleep and Breathing https://doi.org/10.1007/s11325-021-02529-3}

Figure $2 \mathrm{~B}$ in the original version of this article is not correct. The correct figure is shown below. The authors wish to apologize for this error.

The original article has been corrected.

The original article can be found online at https://doi.org/10.1007/ s11325-021-02529-3

\section{Qinghua Liu}

15280096007@163.com

1 Department of Critical Care Medicine, Fujian Provincial Hospital South Branch, The Shengli Clinical Medical College of Fujian Medical University, Fuzhou, Fujian Province, China

2 Department of Respirology, Peking University People's Hospital, Beijing, China

3 Department of Pharmacy, Fujian Provincial Hospital South Branch, The Shengli Clinical Medical College of Fujian Medical University, Fuzhou, Fujian Province, China

4 Department of Otorhinolaryngology, Fujian Provincial Hospital, The Shengli Clinical Medical College of Fujian Medical University, Fujian Medical University, 134 Dongjie Street, Fuzhou, Fujian Province, China 


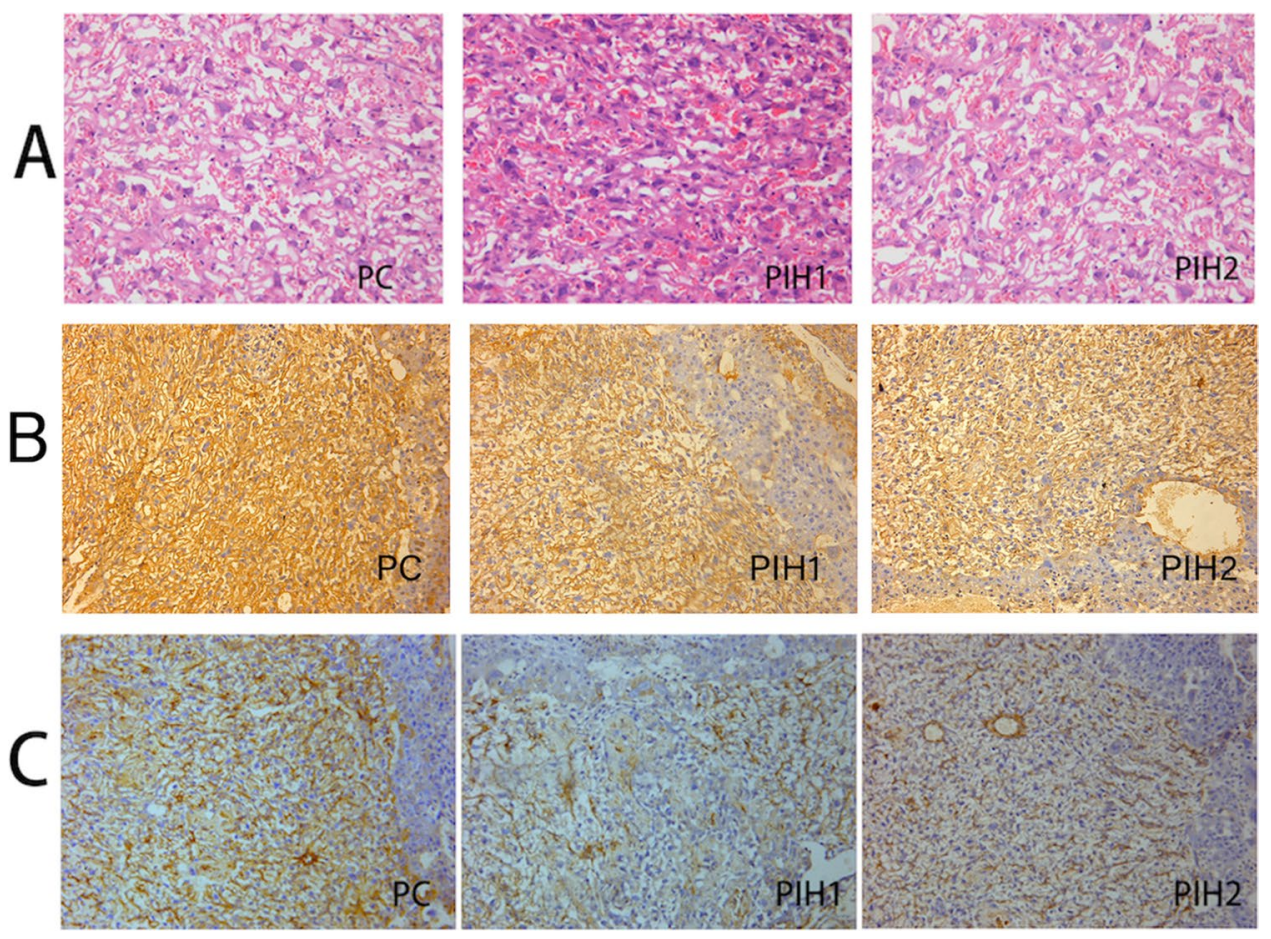

Fig. 2 The histology of placenta, the immunohistochemical staining for CD31, and $\alpha$-SMA of placentas on day 19 in pregnant mice. A The histology of placenta, B the immunohistochemical staining for CD31, and $\mathbf{C} \alpha$-SMA of placentas on day 19 in pregnant mice. HE (hematoxylin and eosin) staining of placenta in pregnant mice $(10 \times$ 20 magnification) showed that microvessels formed irregular webs, least in number, and intravascular agglutination of red blood cells in the placenta of PIH1 group. The placenta in PC group had a denser microvessel network than that in PIH1 group. CD31 immunostaining

Publisher's note Springer Nature remains neutral with regard to jurisdictional claims in published maps and institutional affiliations. of placenta: $(10 \times 10$ magnification $)$. CD31-positive microvessels in the placentas of PC group were greatest in number and finely distributed, while in PIH1 group, CD31-positive microvessels were least in number and irregularly distributed. $\alpha$-SMA immunostaining of placenta: $(10 \times 10$ magnification $) . \alpha$-SMA-positive-microvessels in the placentas of PC group were greatest in number and finely distributed, while in PIH1 group, $\alpha$-SMA-positive-microvessels were least in number and irregularly distributed 